Document downloaded from:

http://hdl.handle.net/10251/176999

This paper must be cited as:

López-Núñez, E.; Pérez Quiles, MJ.; Fajardo, P.; Hoyas, S. (2020). Effect of the horizontal aspect ratio on thermocapillary convection stability in annular pool with surface heat dissipation. International Journal of Heat and Mass Transfer. 148:1-8.

https://doi.org/10.1016/j.ijheatmasstransfer.2019.119140

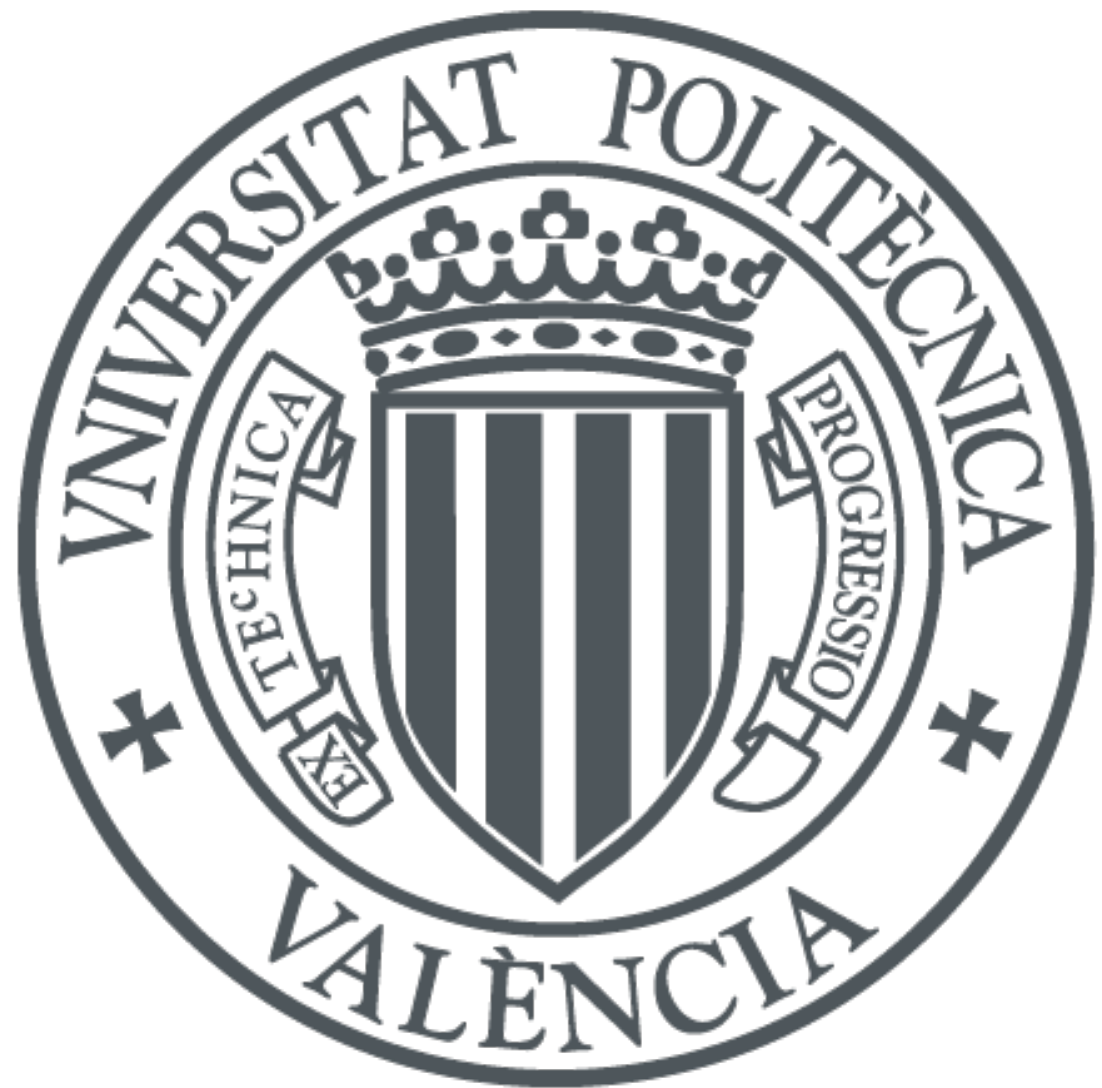

The final publication is available at

https://doi.org/10.1016/j.ijheatmasstransfer.2019.119140

Copyright Elsevier

Additional Information 


\title{
Effect of the Horizontal Aspect Ratio on thermocapillary convection stability in annular pool with surface heat dissipation
}

\author{
E. López-Núñez ${ }^{\mathrm{a}}$, M.J. Pérez-Quiles ${ }^{\mathrm{b}}$, P. Fajardo ${ }^{\mathrm{c}, *}$, S. Hoyas ${ }^{\mathrm{b}}$ \\ ${ }^{a}$ IDR/UPM, Universidad Politécnica de Madrid, Pza. del Cardenal Cisneros 3, Madrid \\ 28040, Spain. \\ ${ }^{b}$ Instituto Universitario de Matemática Pura y Aplicada, Universitat Politècnica de \\ València, València 46022, Spain \\ ${ }^{c}$ Equipo de Propulsión Espacial y Plasmas, Universidad Carlos III de Madrid, Leganés \\ 28911, Spain.
}

\begin{abstract}
A linear stability analysis of the thermoconvective problem of a thin liquid film contained in an annular domain has been conducted. The influence of the horizontal aspect ratio on the solution has been considered by keeping a fixed external wall while the internal radius of the annular domain was modified. The parameter used in the study, $\Gamma_{h}$, has been defined as the ratio of the internal radius to the domain depth. The other control parameter of the study is the Prandtl number ranging from 0.7 to 50, i.e. characteristic of fluids as air to $n$-butanol. The study has been performed for different Bond (Bo) regimes ranging from 0.0 for surface tension dominated flows to 67 for buoyancy dominated ones. Three different kind of bifurcations are found in the $\Gamma_{h}-$ Pr plane for large Bonds, while for low Bonds only two of them appear. In the case of pure buoyancy or surface tension flows, for every $\Gamma_{h}$ there exists a Prandtl number such that oscillatory and stationary coexist in a co-dimension two bifurcation point. These transitions show a strong dependency with the Bond number. Indeed, the lower transition disappears for low Bo and the upper one disappears with intermediate Bo values. Furthermore, there is a non-linear dependency of the number of structures of the growing bifurcation with $\Gamma_{h}$. These co-dimension two lines show a strong dependency with Bo. Firstly, looking at the frontier between HWI and LR
\end{abstract}

\footnotetext{
*Corresponding author. e-mail: pablo.fajardo@uc3m.es
} 
regions, for large Bo numbers, Pr increases with $\Gamma_{h}$, while for low Bo the trend is reversed. Additionally, this transition only appears in the extreme Bo cases, for the central values of the considered, no transition is found. Similarly, the second transition found only appears for Bo larger than 30.

Keywords: Thermocapillary convection, Linear stability, Flow perturbations, Bond number

\section{Introduction}

The topic of thermoconvective flows driven by surface heat dissipation has been widely studied [1, 2, 3] for more than a century. It is well-known that thermoconvective instabilities in fluid layers are caused by two effects: gravity and capillarity forces. Bénard-Marangoni (BM) problem, is the one in which buoyancy does not play a significant role, while when buoyancy is dominant the problem is known as Rayleigh-Benard. If both effects are important, the problem is a hybrid buoyancy-thermocapillary convection. The practical importance of these problems is nowadays widely recognized since it appears in a great variety of processes as: the flow inside distillation columns, crystal growth, film coating processes or droplet and liquid film evaporation [4].

In the most classical BM problem [3], heat is uniformly applied from the bottom wall leading to the emergence of a multicellular pattern. Such flow instabilities were verified experimentally already in 1956 by Block [5], demonstrating the role of surface tension in the formation of this type of flow. If the temperature gradient is parallel to the free interface, then the flow is generally referred to as Marangoni or thermocapillary convection. For these cases, for a relatively small temperature gradient, the flow is two-dimensional (2D) axisymmetric and steady. If the temperature difference is sufficiently increased, however, a flow instability can occur and produce transition to 3D oscillatory states.

A general description of this problem can be found in several works appearing in the literature. Gollub and Benson [6] reported four different transition mechanisms by using Laser Doppler Velocimetry. Smith and Davis [7] predicted, using linear stability analysis, the appearance of the hydrothermal wave instability in an infinite fluid layer in micro-gravity. Latter, Smith also analyzed the influence on the Prandtl number (Pr), reporting two different 
instability mechanisms corresponding to spanwise (for low Pr) or streamwise (for larger Pr) traveling hydrothermal wave (HW) [8].

The instabilities can be triggered, among other processes, by including the effect of a rotation domain [2, 9, 10], flow mixtures with Soret effect [11] or phase change [12]. Imposing a basic dynamic flow through horizontal temperature gradients [13, 14, 15, 16] is one of the most studied effects. The present work is focused in this last aspect.

The onset of instabilities in thermoconvective problems has been analyzed considering a rectangular domain [17], annular geometries [11, 18], of infinite liquid films [19]. There has been some initiatives to develop a theoretical framework of the problem, see[20] and references therein, but for the moment it seems that more effort is needed to develop more powerful mathematical tools to fully understand the process.

In the case of annular pools, Zhang et al. [1, 21, 22] developed a series of three-dimensional simulations to assess the effect of surface heat dissipation on the flow convection on a shallow annular pool fluid at a moderate Prandtl number. The problem has been also studied in an annular geometry but neglecting the heat transfer between the fluid and the atmosphere and considering conduction through the lateral walls of the cylinder [4, 23, 24]. There are also works dealing with localized heating [25], or containers heated by a non-uniform flux [26]. Zhu et al. [27] discussed the relationship between oscillatory frequency and Marangoni number. The effects of gravitation in thermoconvective instabilities has been studied by several authors [28, 29, 30].

The problem considered in the present work is a hybrid buoyancy-thermocapillary convection induced by an inclined temperature gradient. Note that, even if the flow is heated from the bottom wall, it is considered inclined due to the heat exchange between the liquid and the external gas at the free surface. The computational method used is similar to the one described in [31] and is capable of assessing fluids with Prandtl numbers close to unity. The method was validated by comparing the numerical results with the experimental results by Garnier et al. [16. In 32, the authors studied the appearance of co-dimension three bifurcations in the Prandtl-Biot plane and predicted a new kind of instability, the hydrothermal wave of second kind (HWII).

As previously mentioned, depending on the symmetries of the growing perturbation, up to six different competing solutions for the different wave numbers have been identified, namely:

- stationary rolls (SR), similar to the ones of the basic state [7], 
- hydrothermal wave of the first kind or oblique traveling waves (HWI) [7],

- longitudinal rolls (LR) [15],

- standing hydrothermal wave of second class or flower-like wave (HWII) [16, 33],

- two new kinds of hydrothermal waves recently reported by Hoyas et al. [34] for deeper annular domains.

The effect of the domain depth to horizontal dimension ratio $(\Gamma)$ on the onset of the flow motion and the dynamics of the different bifurcations appearing has been studied in [34, 35]. However, to the knowledge of the authors, there has not been any study assessing the influence of the horizontal aspect ratio of the annular domain on the development of flow instabilities, and thus it is the main focus of the present work. The study is performed by varying the internal radio of the cylinder.

The understanding of this flow behavior will contribute to control these instabilities. To achieve this goal, a linear stability analysis, similar to the one in [15], will be performed, but focusing on understanding the effect of the domain horizontal geometry, characterized by the horizontal aspect ratio. This study will be performed in a broad range of Bond numbers, from buoyancy dominant ones to thermocapilary flows.

This manuscript is structured as follows: first a description of the governing equation and the main parameters of the problem are introduced. Then, the problem formulation and the computational domain are presented. The numerical method is described in the third section, and in the fourth one the results are discussed. Finally, the conclusions are presented.

\section{Problem formulation and computational domain}

A sketch of the physical domain modeled in this work is presented in Figure 1. Briefly, the flow is contained in an annular horizontal domain of depth $d$ along the axial coordinate, $z$, and inner and outer radii $a$ and $a+\delta$ (in the $r$ coordinate).

The bottom surface of the annular domain is considered to be rigid and it is heated with a linearly decreasing (with the radius) temperature distribution. The horizontal temperature difference has a value of $T_{G}=2 \mathrm{~K}$, which 


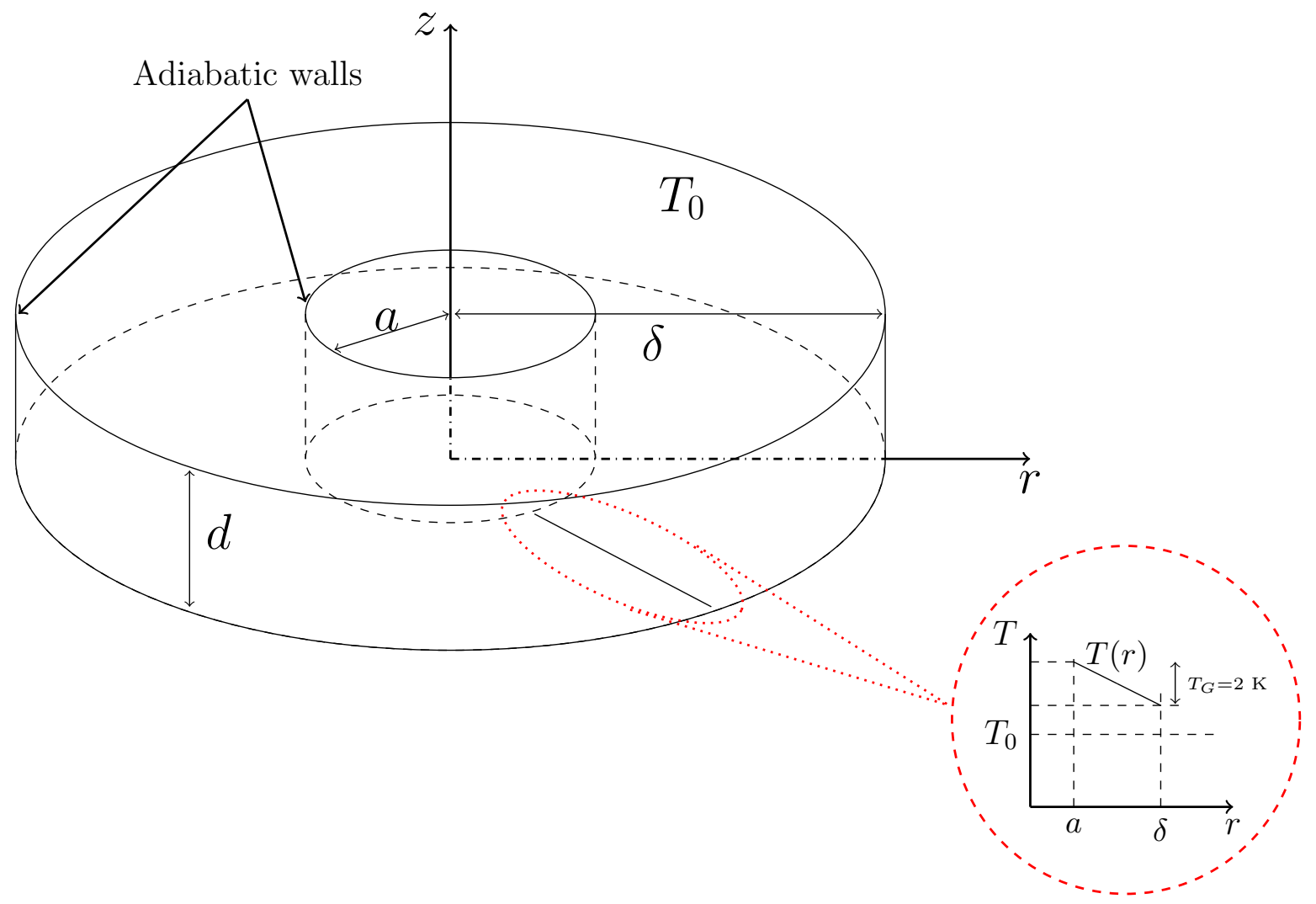

Figure 1: Sketch of the geometry. Lateral walls are considered adiabatic. The fluid is heated from below and the top surface is open to the atmosphere 
is kept constant throughout the study. The two lateral walls of the cylinder are considered rigid and adiabatic. Finally, the top surface of the annular domain is open to the atmosphere. There, the heat transfer between the fluid and the atmosphere (imposed to be at $T_{0}$ ) is modeled through the Biot number, defined in the following section. For the simulations presented in this work the domain depth, $d$, has been set to $2.5 \cdot 10^{-3} \mathrm{~m}$, the external radius, $\delta$, to $2.0 \cdot 10^{-2} \mathrm{~m}$ and the internal radius, $a$ is varied in the range $1 \cdot 10^{-4} \mathrm{~m}$ to $1.99 \cdot 10^{-2} \mathrm{~m}$. In previous works by the authors as 30, 32, the horizontal aspect ratio, $\Gamma_{h}=a / d$, was set to 4 , and the depth aspect ratio $\Gamma=\delta / d$ was set to 8. In [34, the influence of the aspect ratio was assessed sweeping $\Gamma$ in the range $1-8$, and keeping $\Gamma=2 \cdot \Gamma_{h}$, but, as previously mentioned, no study of the horizontal aspect ratio is found. In the present work, the value of $\Gamma_{h}$ ranges between 0.04 to 7.95 .

The fluid layer evolves as described with the flow continuity, momentum and energy conservation equations. The equations are non-dimensionalized as in [13. The set of parameters used are $\kappa$, which is the flow thermal diffusivity, $d$ the characteristic length, $d^{2} / \kappa$ the characteristic time and $\Delta T$ the characteristic temperature, defined as the temperature difference between the bottom plate and the atmosphere. $\Delta T$ is also used as reference temperature in the definition of the Rayleigh and Marangoni numbers. The equations become, respectively

$$
\begin{aligned}
\nabla \cdot \mathbf{u} & =0 \\
\partial_{t} \mathbf{u}+(\mathbf{u} \cdot \nabla) \mathbf{u} & =\operatorname{Pr}\left(-\nabla p+\nabla^{2} \mathbf{u}+\operatorname{Ra} \Theta \mathbf{e}_{z}\right) \\
\partial_{t} \Theta+\mathbf{u} \cdot \nabla \Theta & =\nabla^{2} \Theta
\end{aligned}
$$

In this sort of buoyancy-driven flow problems, the Boussinesq approximation [36] is generally used, as shown in the last term of equation (2). The approximation considers only vertical changes in density affects the movement of the flow, while the effect of small horizontal changes in density can be neglected. Boundary conditions (BCs) are similar to those of references [32, 34. They are summarized in Table 1. As a summary, the bottom plate and the lateral walls are considered as non-slip, i.e. zero velocity. As previously described, the top surface is open to the atmosphere, and thus the thermocapillarity forces are modeled trough the Marangoni condition [13], whereas the heat exchange to the atmosphere is modeled using the Biot number. 
Table 1: Boundary conditions.

\begin{tabular}{|l|l|l|}
\hline$z=0$ & $z=d$ & $r=a, \delta$ \\
\hline \hline$u_{r}=0$ & $\partial_{z} u_{r}+\mathrm{Ma} \partial_{r} \Theta=0$ & $u_{r}=0$ \\
$u_{\phi}=0$ & $r \partial_{z} u_{\phi}+\mathrm{Ma}_{\phi} \Theta=0$ & $u_{\phi}=0$ \\
$u_{z}=0$ & $u_{z}=0$ & $u_{z}=0$ \\
$\Theta=\Delta T-\left(T_{G} / \delta\right) r$ & $\partial_{z} \Theta+\Theta=0$ & $\partial_{n} \Theta=0$ \\
\hline
\end{tabular}

In the previous set of equations (1), (2), and (3) are expressed in cylindrical coordinates and $\mathbf{e}_{z}$ is the unit vector in the axial $(z)$ direction. The variables $u_{r}, u_{\theta}$ and $u_{z}$ are the non-dimensionalized velocity components, $\Theta$ is the non-dimensionalized temperature, and $p$ is the pressure. In the previous equations and BCs described in Table 1, the following set of dimensionless numbers are used:

1. The Prandtl number, Pr, is the ratio of momentum to thermal diffusivity: $\operatorname{Pr}=\nu / \kappa$. In this paper, the $\operatorname{Pr}$ number is varied in the range 0.7-50.

2. The Biot Number, Bi, accounting the heat exchange between the fluid and the atmosphere. This number has been fixed to 0.7 in this study.

3. The Marangoni number, Ma, models the surface tension effects in the top open surface: $\mathrm{Ma}=\gamma_{T} \Delta T d / \rho \kappa \nu$

4. The Rayleigh number, Ra, is representative of the buoyancy effect and it is the control parameter used in this work to trigger the instabilities: $\mathrm{Ra}=g \alpha \Delta T d^{3} / \kappa \nu$

5. The Bond number, Bo, defined as the ratio of Rayleigh to Marangoni numbers, and thus represents the buoyancy against surface tension (thermocapillarity) effects: $\mathrm{Bo}=\frac{\rho g \alpha d^{2}}{\gamma_{T}}$.

In the previous definitions, $\gamma_{T}$ represents the rate of change of surface tension with temperature; $\rho, \alpha$, and $\nu$, are the density, the thermal expansion coefficient, and the kinematic viscosity of the considered fluid, respectively. Finally, $g$ is the gravitational acceleration. It is necessary to clarify to avoid confusions that the Bond number used here is the thermal Bond number defined as the ratio between thermogravitational effects and thermocapillarity effects, and not the Bond number used in colloids, bubbles, and other interfacial systems. Eleven different equispaced Bond numbers are used, ranging from buoyancy dominant flows, Bo $=67$ to thermocapilary ones, Bo $=0$. 


\subsection{Numerical method}

The code used in the present work was implemented in Fortran90, and it is based on a earlier implementation developed by Hoyas et al. 13] for $\operatorname{Pr}=\infty$. The numerical procedure was validated by comparison against experiments in [15]. The generalization to $\operatorname{Pr}<50$ was validated in [31], using the same procedure as in [14], and thus ensuring the validity of the results.

As soon as a temperature gradient is imposed, the fluid evolves reaching a non-time dependent convective motion state known a basic state. Since the domain is cylindrical and the flow is in laminar regime, the solution is basically 2D axisymmetric, and all the derivatives with the angular coordinate, $\phi$, can be neglected. Therefore, the previous equations in cylindrical coordinates become:

$$
\begin{aligned}
r^{-1} \partial_{r}\left(r u_{r}\right)+\partial_{z} u_{z} & =0 \\
\operatorname{Pr}^{-1}\left(u_{r} \partial_{r} u_{r}+u_{z} \partial_{z} u_{r}\right) & =-\partial_{r} p+\Delta_{c} u_{r}-\frac{u_{r}}{r^{2}} \\
\operatorname{Pr}^{-1}\left(u_{r} \partial_{r} u_{z}+u_{z} \partial_{z} u_{z}\right) & =-\partial_{z} p+\Delta_{c} u_{z}+\operatorname{Ra} \Theta \\
u_{r} \partial_{r} \Theta+u_{z} \partial_{z} \Theta & =\Delta_{c} \Theta
\end{aligned}
$$

where $\Delta_{c}=r^{-1} \partial_{r}\left(r \partial_{r}\right)+\partial_{z}^{2}$ is the cylindrical Laplacian operator applied to the axisymmetric domain. The solution of equations (4-7) with the boundary conditions presented in Table 1 can be obtained through different methods, been one of the more used the collocation spectral method [37. To apply this procedure, a Chebyshev expansion of all the flow variables is performed as:

$$
X^{i}(r, z) \simeq \sum_{n=0}^{N} \sum_{m=0}^{M} a_{n m}^{i} T_{n}(r) T_{m}(z)
$$

where $X^{i}$, with $i=1, \ldots, 4$ represent the four different flow variables, i.e., $p, u_{r}, u_{\theta}$ and $\Theta$, respectively, and $T_{j}(x)$ are the Chebyshev polynomial of the first kind of degree $j$. In this formulation, the coefficients of the expansion

$a_{n m}^{i}$ define the value of the flow variable and are now the unknowns of the problem.

The system of equations to solve for the $a_{n m}^{i}$ coefficients is obtained by expanding the flow variables, using Eq. (8), in the flow equations (4.7) and in the boundary conditions of Table 1 ; and evaluation the resultant expression 
in the Chebyshev-Gauss-Lobatto (CGL) points [38]. These CGL points are of interest in boundary problems since they tend to concentrate there, thus improving the accuracy of the method. The order of the method corresponds to the number of points in radial and axial direction correspondingly.

Several authors as [39] have reported the arising of spurious modes due to an improper setting of the boundary condition of pressure. Mancho et al. 40] proposed a procedure based on projecting the equations on the direction normal to the boundaries. The pressure field is then defined except for an arbitrary selected additive constant fixed in a boundary point.

The non-linear terms of the problem are solved by using an iterative Newton-like procedure. As starting solution either a known basic state "close" to the new one or the solution of the linearized set of equations (neglecting the non-linearity in Eq. (5) and Eq. (6)) can be used. In the simulations in the present work, convergence was found in less than 20 iterations.

In the numerical simulations performed in this work, the basic state is stable for low Ra. As Ra is increased the basic state becomes unstable and different bifurcations arise. The shape and type of the appearing solution is complex, and all the parameters of the problem play a determinant role, but in some cases a relationship has been found.

The linear stability analysis is used to determine the threshold for critical parameters of the problem and to identify the type of the growing instabilities. The procedure to perform the stability analysis consists on perturbing the basic state solution with 3D perturbation fields. Since the problem is axisymmetric, and thus periodic in the $\phi$ direction, the flow magnitudes of the perturbation field can be expanded in Fourier modes in the azimuthal direction as:

$$
X(r, \phi, z, t)=X_{b}(r, z)+X_{p}(r, z) e^{i k \phi+\lambda t}
$$

where the subscripts $b$ and $p$ denote the basic state and the induced perturbation fields, respectively. Note again that the basic state does not depend on $\phi$. In the exponential from eq. (9), the wave number of the perturbation is $k \geq 0$ is the wave number, and $i=\sqrt{-1}$ is the imaginary unit.

The stability or eigenvalue problem is solved by introducing the Fourier expansion of the flow variables (eq. (9)) into the general flow equations. The eigenfunction of the problem are determined by the coefficients $a_{n m}^{i}$. After using this procedure, the system of equations is linearized by neglecting 


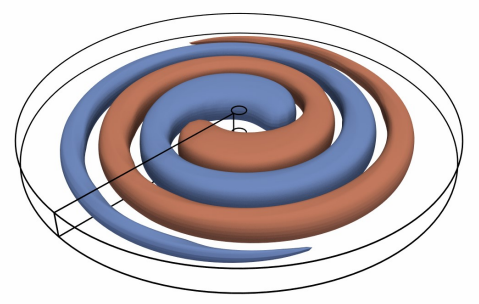

(a)

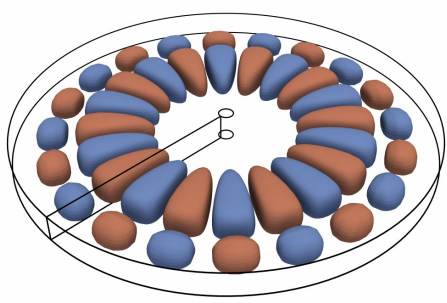

(b)

Figure 2: Three-dimensional plot of the temperature fields of the growing perturbation for $\Gamma_{h}=0.1$ and $\mathrm{Bo}=0$ : hydrothermal waves of first class HWI (a) and longitudinal rolls (b)

the non-linear terms, and thus, a generalized eigenvalue problem is found: $[A] \bar{X}=\lambda[B] \bar{X}$. Due to the boundary conditions, the matrix $[B]$ becomes singular, and thus some of the eigenvalues are infinite. To solve this issue, a transformation proposed by Navarro et al. [41] is used.

The real part of the eigenvalue, $\lambda$, characterizes the instability as it represents the evolution of the perturbation with time. The perturbation decreases, and thus the solution is stable, for negative values of the eigenvalue, $\lambda<0$, and the solution is unstable in any other situation. In this latter case, the imaginary part determines whether the bifurcation is stationary $(\operatorname{Imag}(\lambda)=0)$ as the one shown in Fig. 2a, or oscillatory $(\operatorname{Imag}(\lambda) \neq 0)$ as the one of Fig. 2b.

\subsection{Description of the simulations performed}

The main parameters of the present study, describes before, are summarized in the following Table 2 ,

For each of the simulations presented in this work, Bo, $\operatorname{Pr}$ and $\Gamma_{h}$ are fixed to some values. $\Gamma_{h}$ has been discretized using 170 equispaced points in the range, while 11 values have been used to discretize the Bond number. The discretization of the Pr follows roughly an exponential or geometric growing using 60 points, to ensure a large concentration of points for small and large values of this parameter. Once the Bond number is fixed, both Rayleigh and Maragoni number grow at the same pace. The algorithm used is able to compute the critical Rayleigh (or equivalently the critical Marangoni number) in a few iterations without human interaction. Usually 4 or 5 iterations is 
Table 2: Parameters of the study

\begin{tabular}{|c|c|}
\hline Parameter & Value \\
\hline \hline$\Gamma_{h}$ & $0.04-7.95$ \\
$\Gamma$ & 8 \\
$\mathrm{Pr}$ & $0.7-50$ \\
$\mathrm{Bi}$ & 50 \\
$\mathrm{Bo}$ & $0-67$ \\
$\mathrm{Ra}$ & $0-3000$ \\
$\mathrm{Ma}$ & $0-270$ \\
\hline
\end{tabular}

enough to obtain it. 


\section{Results and discussion}

The variation of neither Bo nor $\operatorname{Pr}$ affects the shape of the basic state significantly, being $\Gamma_{h}$ the most influencing parameter due to the adaptation of the flow regime to the shape of the container. In Fig. 3, the temperature profiles and velocity diagrams for two cases at Bo $=67$ (Fig. 3a) and Bo $=0$ (Fig. 3b) are shown. Figure $3 \mathrm{a}(\mathrm{Bo}=67)$ is similar to those previously reported for $\operatorname{Pr}=\infty$ both experimentally and numerically [15, 30, 34, 42 , and qualitatively similar to those obtained by Riley and Neitzel [20]. The main difference in the velocity diagram between both cases of Fig. 3 is that it presents one $($ for $\mathrm{Bo}=67)$ to three long rolls $(\mathrm{Bo}=0)$. The large roll obtained in the $\mathrm{Bo}=67$ case distribute the heat in convective way, supported by the coupling between the energy and momentum equation trough the Rayleigh term (last term of equation (2)). For $\mathrm{Bo}=0$ convective terms are not longer important, and Marangoni effects are dominant, thus the temperature profile becomes almost horizontal. This has an influence in the velocity field, and the long roll is splitted into three smaller ones. On the contrary, in the $\mathrm{Bo}=67$ case, the temperature profile is largest in the bottom left corner, decreasing to the upper right one. However, return flow regime, characterized by a change in sign in the temperature gradient, as the one described in [34], has not been found in the present study for any combination

of parameters. This basic state showed a great influence in the shape of the growing perturbations. That regime is characterized by the presence of strong negative vertical temperature gradients caused by thermocapillarity, and changing completely the shape of the growing perturbation. At the view of these results, we can safely state that the perturbations described hereinafter do not show any clear dependence coming from the basic state.

For every combination of parameters, horizontal aspect ratio $\Gamma_{h}$, and Bond and Prantdl numbers, the fluid is heated from below until the flow becomes unstable and different bifurcations arise. A mesh size convergence study has been performed to ensure that the number of points used to discretize the computational domain was enough. Figure 4 shows the results of the problem for one of the considered cases for different grid sizes. The number of points in $r, N_{r}$ is varied from 21 to 41 and $N_{z}$ from 13 to 25. The figure shows how all the curves collapse into a single curve and the error is smaller than the fourth decimal position. Therefore, the mesh used has 13 points in $z$ and 27 points in $r$.

The type of the growing perturbation can be seen in Fig. 5, where the 

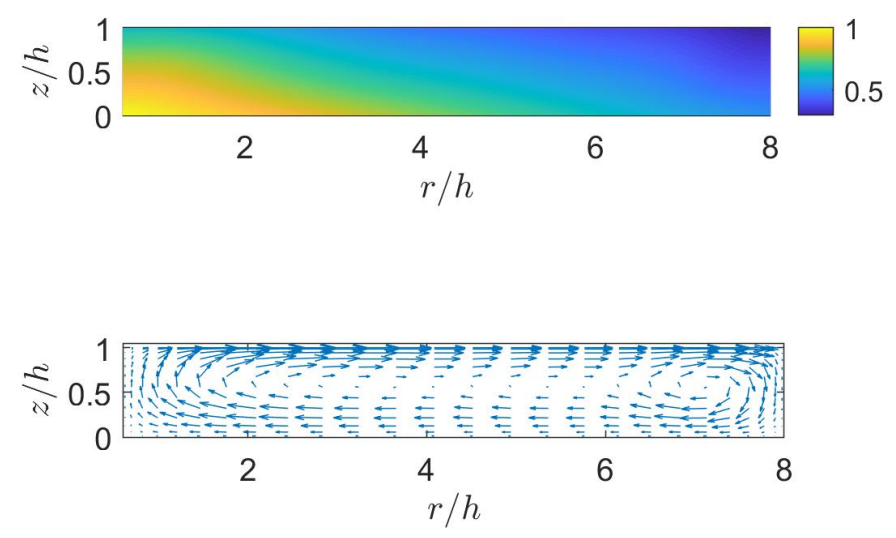

(a)
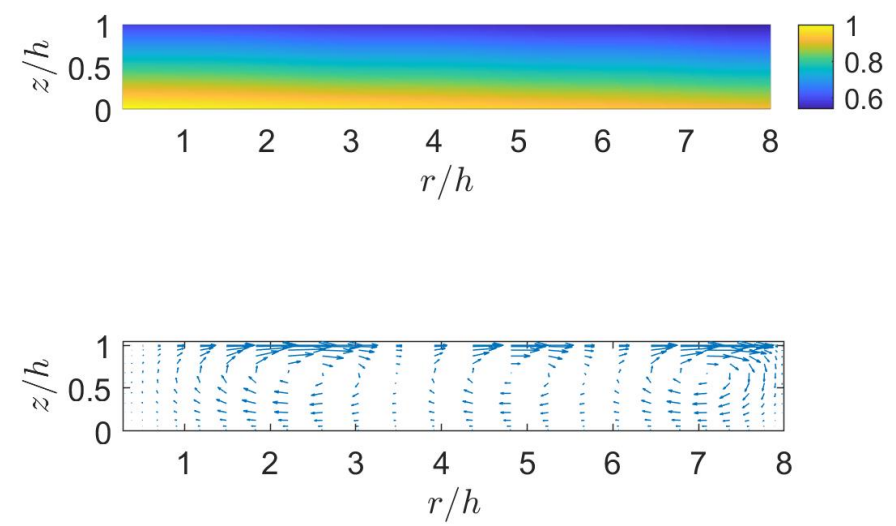

(b)

Figure 3: Temperature profile and velocity diagram of the basic state at their relative critical Rayleigh number for (a) $\mathrm{Bo}=67$ and (b) $\mathrm{Bo}=0$

different regions found in the $\Gamma_{h}-\operatorname{Pr}$ plane are presented. The plots include also the value of the critical wave number $k$ of the perturbation. As it was previously stated, the study has been performed for a range of $\Gamma_{h}=$ 


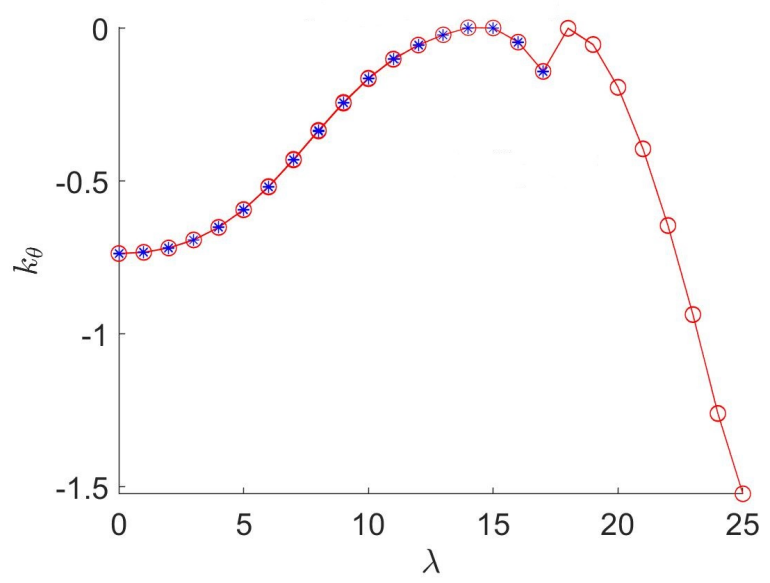

Figure 4: Results of the grid independence study in the results of the problem. All the curves collapse into a single one. Stars represent an oscillatory bifurcation while empty dots do for a stationary one

$0.04-7.95$. The curves separating these regions, indicated with black dots in the figure, are made up of codimension-two points. These points are characterized for having two competing perturbation types, i.e. two critical wave numbers, for the same value of the parameters. Figure 5a shows that three different regimes appear for the largest Bond number studied, Bo $=67$. The bottom region (marked with a I) corresponds to $k=0$. This region presents stationary rolls (SR) with azimuthal symmetry. The boundary of this region appears as an almost horizontal line at approximately $\operatorname{Pr}=1.2$ for all the range of $\Gamma_{h}$ studied. The second region (II) corresponds to cases with complex eigenvalues (oscillatory bifurcation), presenting hydrothermal waves of first class (HWI). Finally, the top region (III) corresponds to cases where a real critical eigenvalue is obtained (stationary bifurcation), i.e. longitudinal rolls. The co-dimension two boundary between these two regions is less welldefined, featuring a kind of sawtooth like behaviour. This can be related to the adaptation of the rolls to the geometry of the container, as it has been recently seen in thermal turbulent Couette flow [43].

Longitudinal rolls (region I) are lost when thermocapilarity is the dominant effect, Fig. 5b, and only regions II and III are found. Transition between these two regions appear for a lower value of Prandtl number in this case. Also, the $\operatorname{Pr}$ value for the co-dimension two line tends to increase with $\Gamma_{h}$ in 5a, while in Fig. 5b it decreases. Furthermore, buoyancy and thermocapi- 


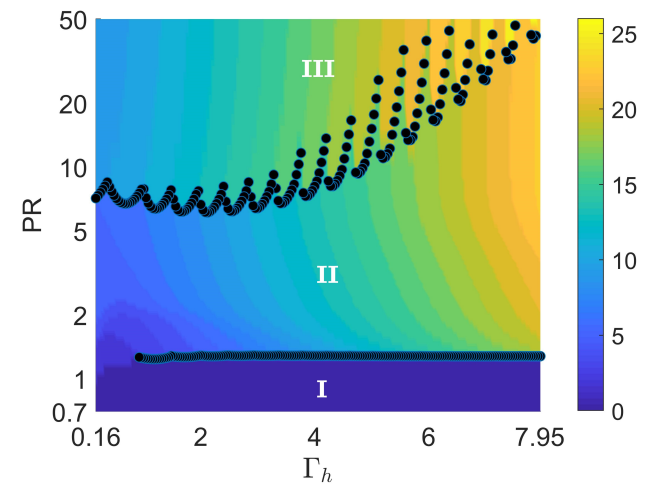

(a)

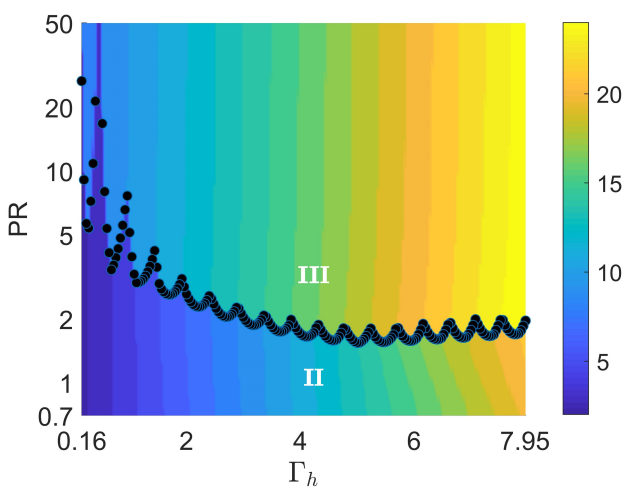

(b)

Figure 5: Critical wavenumber $k$ in the $\Gamma_{h}$-Pr plane for (left) buoyancy, Bo $=67$, and (right) thermocapillarity dominant flows $\mathrm{Bo}=0$. The curves are made up of codimensiontwo points, separating regions with different kinds of growing perturbations

larity favor different effects for the extreme values of $\Gamma_{h}$. The effects in the shape of the growing perturbation for both regimes can be seen in Fig. 6 and Fig. 7, respectively.

Representative top $r-\phi$ plane isotherms for $\mathrm{Bo}=67$, corresponding to the three regions of the $\Gamma_{h}$-Prandt plane of Fig. 5a, are shown in Fig. 6. These plots are indicative of the shape of the growing perturbation. In every row the diameter of the inner cylinder, and thus $\Gamma_{h}$, is fixed (the two extreme values have been considered) and the $\mathrm{Pr}$ is modified along the different columns. This means that along the column, we move through vertical lines in Fig. 5a. The first column shows representative examples of the stationary rolls of region I in the figure, with a wave number equal to 0 . The second and third columns show examples of the hydrothermal waves (HWI) appearing in region II, between Pr 1.2 and approximately 6 (for low $\Gamma_{h}$ ). Finally, in the last column the longitudinal rolls (LR) of region III are shown. The scale of the isotherms of Fig. 6 is normalized using the highest absolute temperature as reference value. Lighter colors indicate hot spots while darker ones represent regions with lower temperature.

Similarly, in Fig. 7 for $\mathrm{Bo}=0.0$, the representative isotherms from the top are now examples of the perturbation that appear in each region of Fig. 5b. The left column show representative HWI for different values of $\Gamma_{h}$, while in the right column different longitudinal rolls are shown. Once again the rows correspond to the two extreme values of $\Gamma_{h}$ considered in the study. 

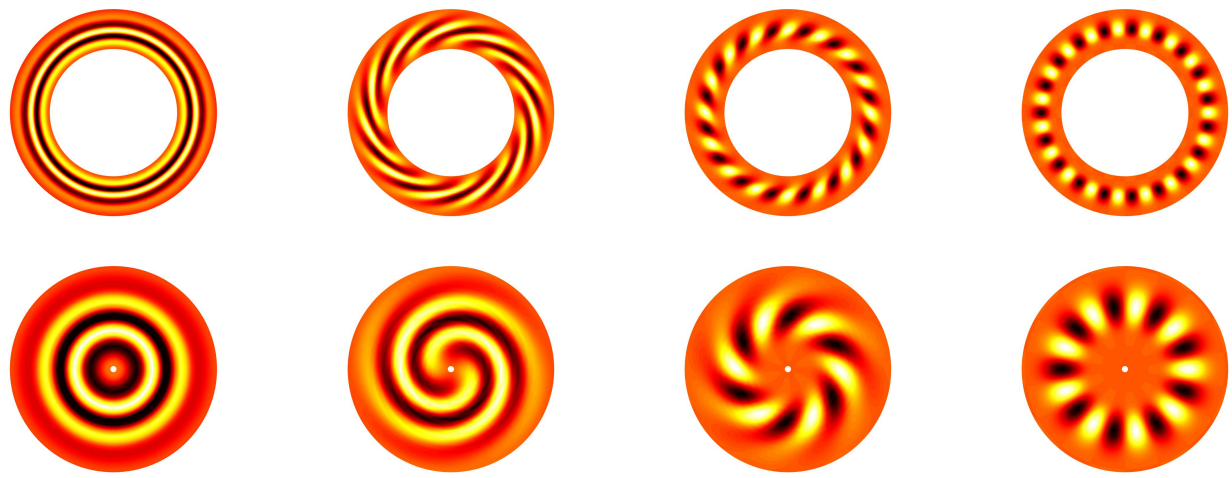

Figure 6: Representative top $r-\phi$ plane isotherms corresponding to the tree regions of Figure 5a. In every row the diameter of the inner cylinder is fixed. The first column shows example of stationary roll. The hydrothermal waves appearing between $\operatorname{Pr} 1$ and 6 appear in the second and third column. Finally, the longitudinal rolls are shown in the last column.
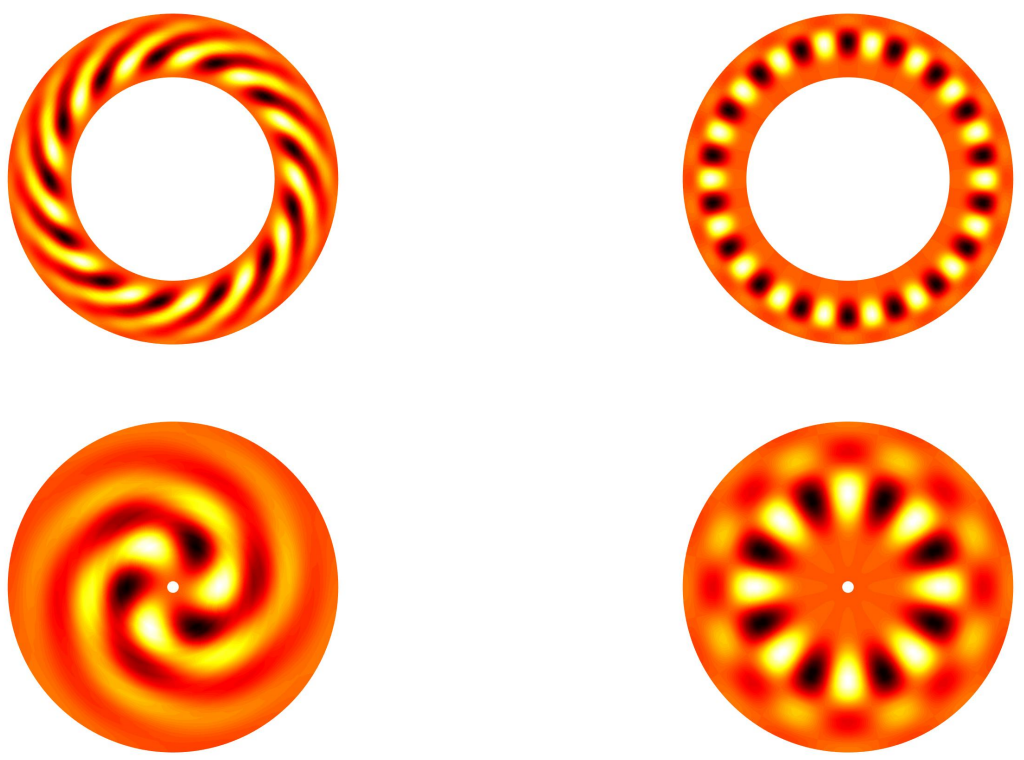

Figure 7: Representative top $r-\phi$ plane isotherms corresponding to the two regions of figure $5 \mathrm{~b}$ for low (top) and large values of $\Gamma_{h}$. In every row the diameter of the inner cylinder is fixed. The first column shows the different hydrothermal waves. Stationary rolls are shown in the last column 
A comparison between Figure $5 \mathrm{a}$ and Figure $5 \mathrm{~b}$ shows that both codimension two curves have a strong dependency on the Bond number. Indeed, the curve I-II totally disappears for $\mathrm{Bo}=0$. As previously said, for each of the Bo values of Figure 8, $n_{d}=170$ points of $\Gamma_{h}$ are considered. For each Bo, and every value of $\Gamma_{h}$, i.e. vertical lines in Fig. 5, we can define the following density function:

$$
\rho_{\mathrm{Bo}}^{\text {case }}\left(\Gamma_{h}\right)= \begin{cases}1 & \text { if there is a codimension } 2 \text { point } \\ 0 & \text { if there is not a codimension } 2 \text { point }\end{cases}
$$

where case can be either the transition I-II or II-III. As there only one codimension point for both types of boundaries, the sum of all cases for a given Bo is

$$
\sum_{j=1}^{n_{d}} \rho_{\mathrm{Bo}}^{\text {case }}\left(\Gamma_{h}(j)\right) \leq n_{d},
$$

and thus, we can define the function

$$
\Lambda^{\text {case }}(\mathrm{Bo})=\frac{\sum_{j=1}^{n_{d}} \rho_{\mathrm{Bo}}^{\text {case }}\left(\Gamma_{h}(j)\right)}{n_{d}} \leq 1 .
$$

This function can be thought as a measure of the number of codimension two points for a particular Bo. A value of $\Lambda^{\text {case }}(\mathrm{Bo})=1$ means that a codimension two point of the considered case (either I-II or II-III) is always found for the considered Bo. The curves obtained for $\Lambda^{I-I I}$ (blue empty dots) and $\Lambda^{I I-I I I}$ (green dots) are presented in figure 8. For every Bo, the Pr ranges from 0.7 to 50. The blue curve is 1 for any Bo larger than 35 . That is, for all the values of $\Gamma_{h}$ considered there is a transition from SR (region I) to HWI (region II) depending on the Prandtl number. However, as Bo is reduced, this transition disappears $(\mathrm{Bo}<20)$ and thus the value of $\Lambda$ becomes zero, meaning that no co-dimensión two points are found for the different $\Gamma_{h}$ and Pr cases. Thus the existence of stationary rolls is totally linked to convective effects (large Bo). This is similar to the results obtained in reference [30], where the stationary rolls were not seen for Bo $<30$.

The green curve in Fig. 8 indicates the transition between hydrothermal waves of the first kind (region II), for small values of $\mathrm{Pr}$, to longitudinal rolls (III), for large values of Pr. In the central region of the figure, i.e. central values of the considered Bo range, the density is almost zero, indicating that there is no transition (or co-dimension two point) and for every 


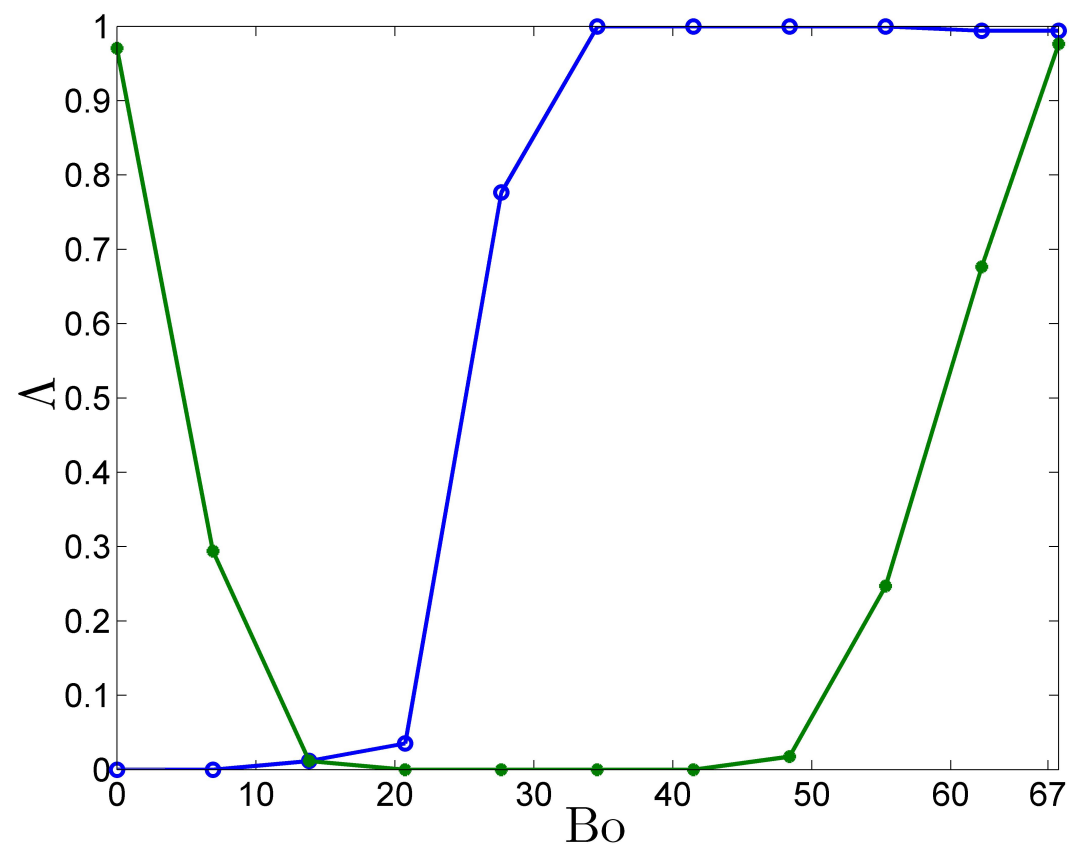

Figure 8: Density of codimension two points as defined in eq. 10$)$, for Pr beween 0.7 and 50. Blue empty circles, stand for $\Lambda^{I-I I}$. Green dots stand for $\Lambda^{I I-I I I}$

Prandtl number and domain aspect ratios, all the bifurcations are originated by a hydrothermal wave (only region II exists in the $\Gamma_{h}$-Pr plane). For the longitudinal rolls region to exist for every $\Gamma_{h}$, it is necessary that the flow would be either thermocapilar (low Bo) or convective (large Bo), but not in a mixed regime. Furthermore, the growing perturbations in both cases are alike, as it can be notice by comparing the second and fourth columns of Fig. 6 with the columns of Fig. 7. This result, together with the ones presented in reference [30] point out at the Biot number as the most important parameter controlling the shape of the growing perturbation, since the main effect of $\Gamma_{h}$ is the change of the number of structures of the growing perturbation.

\section{Conclusions}

In the present work, the influence of the cylindrical domain geometry, in particular the horizontal aspect ratio $\Gamma_{h}$, on the onset of thermoconvective instabilities in a Bernard-Marangoni convection problem is analyzed. In this 
problem, the flow is heated from below with a constant linearly decreasing temperature profile with $2 \mathrm{~K}$ difference between the external and the internal walls. The flow evolves due to this heating until it reaches a basic stationary state.

The control parameters used in this work have been the horizontal aspect ratio, $\Gamma_{h}$ and the Prandtl number. The study has been performed a range of Bond numbers covering the different regimes, i.e., from large Bond, namely $\mathrm{Bo}=67$, in which the flow is dominated by the buoyancy (Rayleigh number); to $\mathrm{Bo}=0.0$ when the problem is dominated by the surface tension (Marangoni number). Two different basic states have been obtained: the first one (for low Bo) presenting several (three) co-rotating rolls and a quasihorizontal temperature gradient, and the second one (for large Bo) featuring a single recirculation in the domain, inducing a condition in which the flow in the central plane is mainly horizontal.

Starting from the basic state, a linear stability analysis is performed to obtain the type and shape of the dominant perturbations. It is concluded that the basic state will not affect the shape of the perturbations. Up to three different perturbations (LR, HWI and SR) have been found in the $\Gamma_{h}$-Prandtl plane for Bo $=67$ and the two transition curves, formed of codimension two points, have been obtained. The boundary between the LR and HWI is a horizontal line for $\operatorname{Pr} \approx 1.2$, while the frontier between HWI and SR presents a sawtooth like behavior with the Pr tending to increase with $\Gamma_{h}$. The critical wave number is 0 for all the values of $\Gamma_{h}$ and low $\operatorname{Pr}$ (SR region) and it increases towards the large $\Gamma_{h}$ and large $\operatorname{Pr}$ (north-east) corner. Notice that trying to reproduce this results experimentally requires using different fluids to cover a wide range of Prandtl numbers assessed (from 0.750 ), and each experiment would need a typical stabilization time of several hours which makes it almost unaffordable. On the contrary, for the $\mathrm{Bo}=0$ case, the critical wave number lines are almost vertical lines. Concerning the perturbations, the region with SR disappears now, and we only find HWI and LR.

The most interesting result is the strong dependency of this transitions (co-dimension two points) with the Bond number. This can be first seen by comparing the trends found in the frontier between HWI and LR in the $\Gamma_{h}$-Pr planes from Fig. 5. While for the larger Bond the Pr tends to increase with $\Gamma_{h}$, for the lower Bond one the behavior is the opposite. However, the influence is even larger. Figure 8 shows that the transition between regions III appears for any domain shape $\left(\Gamma_{h}\right)$ for Bo larger than 30, but it completely 
disappears (density of co-dimension two points is almost zero) for Bo smaller than 20. The transition be between HWI (region II) to LR (region III) only appears for all the $\left(\Gamma_{h}\right)$ in the extreme Bo cases, for the central values of the considered Bo range, the density is almost zero, indicating that there is no transition (or co-dimension two point). This means that for the LR perturbation to exists it requires that the flow is either thermocapilar (low Bo) or convective (large Bo). Another interesting fact is that the growing perturbations in these two cases are quite similar. The results obtained in the present study indicate that the Biot number is the most important parameter affecting the shape of the growing perturbations, while $\Gamma_{h}$ basically modifies the number of structures appearing in the perturbations and thus the value of the critical wave number shown in Fig. 5.

\section{Acknowledgements}

SH and MJPQ work have been supported by project RTI2018-102256-BI00 of Mineco/FEDER. PF work has been partially supported by the Spain's National Research and Development Plan (Project ESP2016-75887) and by the CHEOPS project (Grant Agreement 730135). This work was supported by a generous grant of computer time from the supercomputing center of the UPV.

\section{References}

[1] Li Zhang, You Rong Li, Chun Mei Wu, and Lu Zhang. Effect of surface heat dissipation on thermocapillary convection of moderate prandtl number fluid in a shallow annular pool. Journal of Crystal Growth, 514:21-28, 2019.

[2] Han Ming Li, Wan Yuan Shi, and Michael K Ermakov. Thermocapillary flow instabilities of medium prandtl number liquid in rotating annular pools. International Journal of Thermal Sciences, 120:233-243, 2017.

[3] H. Bènard. Les tourbillons cellulaires dans une nappe liquide. Rev. Gen. Sci. Pure Appl., 11:1261-1271, 1900.

[4] W.Y. Shi, M.K. Ermakov, Y.-R. Li, L. Peng, and N. Imaishi. Influence of buoyancy force on thermocapillary convection instability in the differ- 
entially heated annular pools of silicon melt. Microgravity Science and Technology, 21(SUPPL. 1):S289-S297, 2009.

[5] Myron J Block. Surface tension as the cause of bénard cells and surface deformation in a liquid film. Nature, 178(4534):650-651, 1956.

[6] JoPo Gollub and SV Benson. Many routes to turbulent convection. Journal of Fluid Mechanics, 100(3):449-470, 1980.

[7] Marc K. Smith and Stephen H. Davis. Instabilities of dynamic thermocapillary liquid layer. part 1. convective instabilities. Journal of Fluid Mechanics, 132:119-144, 1983.

[8] Marc K Smith. Instability mechanisms in dynamic thermocapillary liquid layers. The Physics of fluids, 29(10):3182-3186, 1986.

[9] H. F. Bauer and W Eidel. Marangoni convection in an annular cylindrical tank. Heat and mass transfer, 33(5-6):457-464, 1998.

[10] Linmao Yin, Zhong Zeng, Zhouhua Qiu, Huan Mei, Liangqi Zhang, and Yongxiang Zhang. Linear stability analysis of thermocapillary flow in a slowly rotating shallow annular pool using spectral element method. International Journal of Heat and Mass Transfer, 97:353-363, 2016.

[11] Jia Jia Yu, You Rong Li, Chun Mei Wu, and Jie Chao Chen. Threedimensional thermocapillary-buoyancy flow of a binary mixture with soret effect in a shallow annular pool. International Journal of Heat and Mass Transfer, 90:1071-1081, 2015.

[12] PJ Sáenz, P Valluri, K Sefiane, G Karapetsas, and OK Matar. On phase change in marangoni-driven flows and its effects on the hydrothermalwave instabilities. Physics of Fluids, 26(2):024114, 2014.

[13] S. Hoyas, H. Herrero, and A.M. Mancho. Thermal convection in a cylindrical annulus heated laterally. Journal of Physics A: Mathematical and General, 35(18):4067-4083, 2002.

[14] S. Hoyas, H. Herrero, and A.M. Mancho. Bifurcation diversity of dynamic thermocapillary liquid layers. Physical Review E - Statistical, Nonlinear, and Soft Matter Physics, 66(5):057301/1-057301/4, 2002. 
[15] S. Hoyas, H. Herrero, A.M. Mancho, N. Garnier, and A. Chiffaudel. Bénard-Marangoni convection in a differentially heated cylindrical cavity. Phys. Fluids, 1(7):054104, 2005.

[16] N. Garnier and A. Chiffaudel. Two dimensional hydrothermal waves in an extended cylindrical vessel. European Physical Journal B, 19(1):8795, 2001.

[17] Y Li, R Grigoriev, and M Yoda. Experimental study of the effect of noncondensables on buoyancy-thermocapillary convection in a volatile low-viscosity silicone oil. Physics of Fluids, 26(12):122112, 2014.

[18] Jie Chao Chen, You Rong Li, Jia Jia Yu, Li Zhang, and Chun Mei Wu. Flow pattern transition of thermal-solutal capillary convection with the capillary ratio of- 1 in a shallow annular pool. International Journal of Heat and Mass Transfer, 95:1-6, 2016.

[19] Lahcen Bammou, Serge Blancher, Yves Le Guer, Kamal El Omari, and Brahim Benhamou. Linear stability analysis of poiseuille-bénardmarangoni flow in a horizontal infinite liquid film. International Communications in Heat and Mass Transfer, 54:126-131, 2014.

[20] R. J. Riley and G. P. Neitzel. Instability of thermocapillary-buoyancy convection in shallow layers. Part 1. Characterization of steady and oscillatory instabilities. Journal of Fluid Mechanics, 359:143-164, 1998.

[21] Li Zhang, You Rong Li, Chun Mei Wu, and Qiu Sheng Liu. Flow bifurcation routes to chaos of thermocapillary convection for low prandtl number fluid in shallow annular pool with surface heat dissipation. International Journal of Thermal Sciences, 125:23-33, 2018.

[22] Li Zhang, You Rong Li, Chun Mei Wu, and Jia Jia Yu. Surface heat dissipation dependence of thermocapillary convection of moderate prandtl number fluid in an annular pool. Microgravity Science and Technology, pages 1-13, 2019.

[23] L. Peng, Y.-R. Li, W.-Y. Shi, and N. Imaishi. Three-dimensional thermocapillary-buoyancy flow of silicone oil in a differentially heated annular pool. International Journal of Heat and Mass Transfer, 50(56):872-880, 2007. 
[24] W.Y. Shi, X. Liu, G. Li, Y.-R. Li, L. Peng, M.K. Ermakov, and N. Imaishi. Thermocapillary convection instability in shallow annular pools by linear stability analysis. Journal of Superconductivity and Novel Magnetism, 23(6):1185-1188, 2010.

[25] Igor I Wertgeim, Marat A Kumachkov, and Alexander B Mikishev. Periodically excited marangoni convection in a locally heated liquid layer. The European Physical Journal Special Topics, 219(1):155-165, 2013.

[26] Rachid Es Sakhy, Kamal El Omari, Yves Le Guer, and Serge Blancher. Rayleigh-bénard-marangoni convection in an open cylindrical container heated by a non-uniform flux. International Journal of Thermal Sciences, 86:198-209, 2014.

[27] Peng Zhu, Li Duan, and Qi Kang. Transition to chaos in thermocapillary convection. International Journal of Heat and Mass Transfer, 57(2):457464, 2013.

[28] E.R.G. Eckert, R.J. Goldstein, W.E. Ibele, S.V. Patankar, T.W. Simon, T.H. Kuehn, P.J. Strykowski, K.K. Tamma, A. Bar-Cohen, J.V.R. Heberlein, J.H. Davidson, J. Bischof, F.A. Kulacki, U. Kortshagen, and S. Garrick. Heat transfer - a review of 1997 literature. International Journal of Heat and Mass Transfer, 14(43):2431-2528, 2000.

[29] S. M. O'Shaughnessy and A. J. Robinson. Heat transfer near an isolated hemispherical gas bubble: The combined influence of thermocapillarity and buoyancy. International Journal of Heat and Mass Transfer, 62(1):422-434, 2013.

[30] S Hoyas, P Fajardo, A Gil, and MJ Perez-Quiles. Analysis of bifurcations in a benard-marangoni problem: Gravitational effects. International Journal of Heat and Mass Transfer, 73:33-41, 2014.

[31] A.J. Torregrosa, S. Hoyas, M.J Perez-Quiles, and J.M. Mompo-Laborda. Bifurcation diversity in an annular pool heated from below: Prandtl and biot numbers effects. Communications in Computational Physics, 13(2):428-441, 2013.

[32] S. Hoyas, A. Gil, P. Fajardo, and M.J. Pérez-Quiles. Co-dimension Three Bifurcations in a Bénard-Marangoni Problem. Physical Review E, 88(015001), 2013. 
[33] E. Favre, L. Blumenfeld, and F. Daviaud. Instabilities of a liquid layer locally heated on its free surface. Physics of Fluids, 9(5):1473-1475, 1997.

[34] S. Hoyas, , P. Fajardo, and M.J. Pérez-Quiles. Influence of geometrical parameters on the linear stability of a Bénard-Marangoni problem. Physical Review E, 93(4)(043105), 2016.

[35] Jia Jia Yu, You Rong Li, D. F. Ruan, and Chun Mei Wu. Aspect ratio and capillary ratio dependence of thermal-solutal capillary-buoyancy flow of a binary mixture in an annular pool. International Journal of Thermal Sciences, 136:347-356, 2019.

[36] Joseph Boussinesq. Théorie de l'écoulement tourbillonnant et tumultueux des liquides dans les lits rectilignes a grande section, volume 1. Gauthier-Villars, 1897.

[37] Steven A. Orszag. Comparison of pseudospectral and spectral approximation. Studies in Applied Mathematics, 51(3):253-259, 1972.

[38] C. Canuto, M.Y. Hussaini, A. Quarteroni, and T.A. Zang. Spectral Methods in Fluid Dynamics. Springer-Verlag, 1988.

[39] C. Bernardi and Y. Maday. Approximations spectrales des problèmes aux limites elliptiques. Springer-Verlag, 1992.

[40] A.M. Mancho and H. Herrero. Instabilities in a laterally heated liquid layer. Physics of Fluids, 12(5):1044-1051, 2000.

[41] M.C. Navarro, H. Herrero, and S. Hoyas. Chebyshev collocation for optimal control in a thermoconvective flow. Communications in Computational Physics, 5(2-4):649-666, 2009.

[42] S. Hoyas, H. Herrero, and A. M. Mancho. Thermocapillar and thermogravitatory waves in a convection problem. Theoretical and Computational Fluid Dynamics, 18(2-4):309-321, 2004.

[43] F. Alcántara-Ávila, Gandía Barberá, and S. Hoyas. Evidences of persisting thermal structures in couette flows. International Journal of Heat and Fluid Flow, 2019. 